\title{
Función disciplinaria del Autorregulador del Mercado de Valores en Colombia
}

\author{
Disciplinary function of the securities market \\ self-regulator in Colombia
}

https://doi.org/10.15332/iust.v0i17.2421

\begin{abstract}
Camilo E. Quiñónez Avendaño
Abogado, especialista en Derecho Comercial; especialista en Derecho Empresarial; magister en Derecho Económico; docente asociado Universidad Autónoma de Bucaramanga, UNAB Correoelectrónico: equinone@unab.edu.co.
\end{abstract}

\section{Resumen}

El Autorregulador del Mercado de Valores - AMV en Colombia cumple una función disciplinaria que tiene un marco normativo especializado, cuya aplicación busca generar confianza en el mercado y prevenir el riesgo sistémico. En desarrollo de esta función no surge un mecanismo de protección patrimonial a los inversionistas, en tanto no está diseñada para proteger derechos patrimoniales sino para generar confianza en el mercado y para que los intermediarios de valores administren el riesgo reputacional.

Palabras clave: Autorregulación, mercado de valores, función disciplinaria.

\begin{abstract}
The Securities Market Self-Regulator - AMV in Colombia fulfills a disciplinary function that has a specialized regulatory framework, whose application seeks to generate confidence in the market and prevent systemic risk. In developing this function, there is no mechanism for asset protection for investors, as it is not designed to protect property rights but to generate confidence in the market and for securities intermediaries to manage reputational risk.
\end{abstract}

Keywords: Self-regulation, stock market, disciplinary function.

\section{Résumé}

L’autorégulation du marché des valeurs mobilières - AMV en Colombie remplit une fonction disciplinaire dotée d'un cadre réglementaire spécialisé, dont l'application vise à susciter la confiance dans le marché et à prévenir le risque systémique. En développant cette fonction, il n'y a pas de mécanisme de protection des actifs pour les investisseurs, car il n'est pas conçu pour protéger les droits de propriété mais pour générer la confiance dans le marché et pour les intermédiaires en valeurs mobilières pour gérer le risque de réputation.

Mots-clés: Autorégulation, bourse, fonction disciplinaire. 
IUSTITIA 


\section{Función disciplinaria del Autorregulador del Mercado de Valores en Colombia ${ }^{1}$}

Camilo E. Quiñónez Avendaño

\section{INTRODUCCIÓN}

En este documento se presenta una parte del resultado de una investigación jurídica sobre la función disciplinaria del autorregulador del mercado de valores en Colombia. La pregunta que guió el desarrollo de la investigación es: ¿Cómo se concretan los fines que inspiran la intervención estatal en el mercado de valores colombiano en la regulación de la función disciplinaria ejercida por el autorregulador del mercado de valores? Los resultados que se comparten constan de cinco partes: en la primera se presenta la definición de autorregulación como soporte conceptual; en la segunda se describe el desarrollo normativo de la autorregulación en el mercado de valores colombiano; en la tercera se muestran los rasgos distintivos de la función disciplinaria, en la cuarta, la relación con los objetivos de la intervención estatal en los mercados de valores y, finalmente, algunas reflexiones que operan a modo de conclusión que no son un cierre del tema, sino que muestran otros campos de análisis y reflexión que se derivan de la investigación.

Se trata de una investigación jurídica, exploratoria que hace uso de la descripción y el análisis de un modelo de regulación con impacto en el mundo de los negocios, específicamente mercado de valores, en la cual se utilizó el método dialéctico con apoyo en la inducción, deducción, análisis y síntesis.

\section{DEFINICIÓN DE AUTORREGULACIÓN}

En sentido amplio, la autorregulación es un proceso formado por pensamientos auto-generados, emociones y acciones que están planificadas y adaptadas cíclicamente para lograr la obtención de los objetivos personales (Zimmerman, 2000). Bajo esta perspectiva, la autorregulación es una estrategia para alcanzar metas personales y no es un concepto o categoría conceptual propia de los mercados de valores.

Todo comportamiento humano se orienta hacia una meta y esta se mantiene mientras tenemos una expectativa para alcanzarla. En el mercado de valores, el emisor

$1 \quad$ El trabajo que se publica es producto de la investigación titulada "Interpretación teleológica de la función disciplinaria del autorregulador del mercado de valores en Colombia”, radicada ante la Dirección General de Investigaciones de la Universidad Autónoma de Bucaramanga bajo el código I78083. 
tiene como meta obtener financiación, el inversionista busca la mayor rentabilidad y el intermediario de valores busca ganancias por su labor de intermediación entre emisor e inversionista. Bajo estos pilares gira el día a día de los mercados y las relaciones que surgen en él permiten que estas metas o intereses individuales estén en una interacción permanente, en principio, bajo unos pensamientos que les permitan maximizar sus beneficios.

La búsqueda de altos niveles de satisfacción en los mercados de valores, en particular en el colombiano, no permite que los pensamientos, emociones e intereses puedan ponerse en acción sin límites. Existe una intervención externa al sujeto proveniente del Estado que indica cuáles son los patrones que guían y deben guiar las interacciones entre los agentes del mercado. Así, partimos de la teoría estándar de la regulación, dentro de la cual Anthony Ogus señaló que la regulación es el ejercicio del poder colectivo con el propósito de remediar los fallos de mercado para proteger al público del comportamiento monopólico, de la competencia destructiva, del abuso del poder económico privado o del efecto de las externalidades (citado en Larrañaga, 2009, p. 20).

En ejercicio de ese poder colectivo, la autorregulación se ha vinculado a las relaciones entre los sujetos del mercado de valores, donde tomamos como referentes qué es un acuerdo institucional según el cual una organización (privada) regula el comportamiento de sus miembros (Robb Bagott), citado por Rodríguez, 2014), y que es una forma de regulación y disciplina de la conducta por uno mismo, en la que un grupo de personas u organizaciones de manera conjunta, ejecutan funciones de regulación respecto de sus propias actividades y de todas las de aquellos que aceptan su autoridad (Black, 1996).

En su faceta de forma de regulación, basados en Ariza (2012) caracterizamos la autorregulación como:

i) La existencia de normas jurídicas provenientes del consentimiento propio de los interesados, que en esta investigación son los intermediarios de valores, lo que supone un sometimiento voluntario a las disposiciones normativas, pero un cumplimiento obligatorio, una vez se ha decidido someterse.

ii) Las normas con las que se concreta la autorregulación deben ceñirse u obedecer a categorías normativas de orden superior.

iii) El fenómeno jurídico denominado autorregulación comprende la facultad para crear normas o disposiciones con carácter obligatorio; así como la de disciplinar y sancionar a quienes, sometidos a la autorregulación, omitan su deber de cumplir con los mandatos.

iv) La autorregulación es una figura compatible con los mercados de valores, surge de la misma informalidad de los participantes frente al crecimiento de la actividad y la baja regulación estatal, es decir, frente a la necesidad de establecer reglas a las que los miembros debían someterse para mejorar el orden en el mercado. 
v) El mercado financiero está en constante crecimiento y desarrollo que reclama fijar barreras y estándares de conducta en el mercado.

De lo expresado se tiene que, el fundamento de la figura es la autonomía de la voluntad, el consentimiento de los interesados, que bien podrían expedir sus propias normas de conducta o encargar de esta labor al gremio al que pertenecen o a un sujeto de derecho privado. El propósito del sector o gremio que de manera voluntaria se autorregula, será hacer explícito su compromiso con el respeto de los intereses generales de la sociedad, los cuales se materializan por medio de reglas, organización o actividades para el bien común que garanticen un comportamiento aceptable, no solo desde la legalidad sino desde la ética ${ }^{2}$.

La importancia del mercado de valores en la actualidad y su constante crecimiento hace necesaria la existencia de reglas para el mercado de valores provenientes del Estado, y reglas provenientes de los particulares que sean complementarias y sirvan para moldear la conducta de los sujetos, en particular de los intermediarios. Estas normas particulares entran a formar parte del sistema jurídico, solo si el Estado acepta que los intervinientes en el mercado creen sus propias normas y se encarguen de su cumplimiento. Rodríguez Fernández (2014) destaca que en este contexto se hacen visibles conceptos como regulación, autorregulación, corregulación o desregulación, a la vez que reconoce que el mercado de valores no es ajeno a esta realidad y que la autorregulación es eje fundamental del funcionamiento del mismo mercado.

\section{AUTORREGULACIÓN EN EL MERCADO DE VALORES COLOMBIANO}

En Colombia la autorregulación tiene su origen en 1928 con la creación de la Bolsa de Valores de Bogotá. Los agentes del mercado de valores, sin existir regulación sobre el asunto en los estatutos sociales de esta entidad, decidieron crear un mercado organizado mediante la expedición de normas de funcionamiento y la creación de la Cámara Disciplinaria de la Bolsa, a quien se le encargó el juzgamiento de los miembros de la bolsa por el incumplimiento de las normas internas (estatutarias) o por la trasgresión de obligaciones bursátiles y sociales contenidas en los reglamentos de la bolsa, y además se le atribuyó la facultad de imponer sanciones disciplinarias.

Con la expedición de la Ley 964 de 2005, artículos 3 y 24 a 37, la autorregulación pasa a tener un tratamiento legal con mayor cobertura: i. Se menciona de manera expresa como una actividad dentro del mercado. ii. Somete a supervisión del Estado a la entidad que actúe como autorregulador. iii. Impone la necesidad de que quien ejerza la autorregulación sea una entidad constituida o que se constituya en Colombia.

2 Una variable, objeto de análisis, y si la autorregulación se adopta de manera voluntaria o si la normativa estatal obliga a su diseño y al sometimiento a normas de autorregulación. Esta variable no incide en la definición que aquí se expresa. 
iv. Se impone al Gobierno nacional la obligación de expedir normas que desarrollen la autorregulación. v. Fija las reglas de la autorregulación en Colombia.

Del contenido de la citada ley se extraen los siguientes rasgos distintivos de la autorregulación en Colombia:

a) Se le atribuyen funciones normativas, de supervisión y disciplinaria, las que ejerce bajo la supervisión de la Superintendencia de Valores.

b) Impone a quienes ejercen actividades de intermediación la obligación de autorregularse.

c) Autoriza que en Colombia pueden actuar como autorreguladores del mercado de valores las organizaciones que se constituyan exclusivamente para ese fin, las organizaciones gremiales o profesionales, las bolsas de valores, las bolsas de productos agropecuarios, las asociaciones gremiales o profesionales, las bolsas de productos agropecuarios, agroindustriales o de otros comodities, y las sociedades administradoras de sistemas de negociación ${ }^{3}$.

d) Se les impone la obligación de responder por perjuicios que causen por dolo o por culpa en ejercicio de sus funciones.

e) Se impone a la Superintendencia Financiera las siguientes responsabilidades: conceder permiso a quien vaya a ejercer funciones de autorregulación y ejercer la supervisión sobre estos organismos.

f) Se autoriza a la Superintendencia Financiera para que suscriba memorandos de entendimiento con el autorregulador, para coordinar esfuerzos en materia disciplinaria, de supervisión y de investigación.

g) Se fijan requisitos para ejercer la autorregulación.

h) Impone a los organismos de autorregulación los siguientes deberes relacionados con la función disciplinaria:

- Asegurar los mecanismos para el ejercicio de la función disciplinaria, de la aceptación o de rechazo de sus miembros y de la provisión de los servicios del organismo de autorregulación.

- Establecer procedimientos e iniciar de oficio o a petición de parte acciones disciplinarias por el incumplimiento de normas estatales del mercado de valores y normas de autorregulación.

- Le da la competencia para decretar, practicar y valorar pruebas, determinar responsabilidad disciplinaria e imponer sanciones que deben ser de conocimiento de la Superintendencia Financiera.

3 Hasta el momento en que se escribe este artículo, en Colombia solo existe un organismo autorregulador, denominado Corporación Autorregulador del Mercado de Valores de Colombia, creado en el año 2006, la cual fue constituida como una corporación de carácter privado sin ánimo de lucro, de carácter nacional, que se rige por la Constitución, las normas civiles, la Ley 964 de 2005 y las normas que la desarrollan, por sus estatutos y reglamentos y por los principios que se aplican a los organismos de autorregulación. 
- Señala pautas para el diseño del procedimiento disciplinario: formular los cargos, notificar al miembro y dar la oportunidad para ejercer el derecho de defensa.

- Impone que todo proceso disciplinario debe tener como soporte: la conducta realizada por el miembro o persona vinculada, las normas jurídicas vulneradas, la sanción y la razón por la cual se imponga.

i) En relación con a la función normativa, exige que el autorregulador debe expedir un reglamento que debe ser cumplido por las personas sobres las cuales ejerzan sus funciones. Este reglamento debe ser autorizado por la Superintendencia Financiera, es de obligatorio cumplimiento y se presume conocido por quienes se encuentren sometidos a estos.

Conforme a este marco normativo, se percibe que la disciplina de los intermediarios es compartida entre el AMV y la Superintendencia Financiera de Colombia. Para coordinar la labor, las entidades han suscrito documentos que en lenguaje técnico se conocen como "memorandos de entendimiento". El primero de ellos es de fecha 21 de diciembre de 2006 y el segundo de noviembre 9 de 2007, en ambos, el objeto del acuerdo consiste en que el AMV sea el supervisor en primera línea de supervisión, investigación y sanción de las conductas de mercado de los intermediarios de valores. En el segundo de los documentos mencionados se faculta al AMV para ser quien realice la inspección de asuntos relacionados con obligaciones institucionales que deben tener en cuenta las entidades intermediarias de valores respecto a las actividades que ejercen en el mercado: el usar sistemas de grabación de comunicaciones telefónicas, mensajes de datos similares; la prohibición de utilizar teléfonos móviles y otros dispositivos de comunicación en las mesas de negociación y, el incumplimiento de obligaciones relacionadas con al debida administración de conflictos de intereses.

La Superintendencia Financiera de Colombia asumió como responsabilidad la actividad de los intermediarios que tengan impacto sistémico; asuntos de naturaleza prudencial de los intermediarios de valores y el cumplimiento de normativas sobre temas contables, ejercicio irregular de la actividad de intermediación e incumplimiento de normas relacionadas con el sistema de prevención del lavado de activos y financiación del terrorismo.

De otra parte, se advierte que la regulación colombiana no define autorregulación. En la investigación cuyo resultado se presenta en este artículo, se tomó como referente la definición dada por la Corte Constitucional colombiana:

La autorregulación es, entonces, una institución que tiene su fuente originaria en el campo del derecho privado y que encuentra un claro fundamento de principio en la autonomía de la voluntad privada entendida esta como la facultad reconocida por el ordenamiento jurídico positivo a las personas de disponer de sus intereses con efecto vinculante y, por tanto, para crear derechos y obligaciones, con los límites generales del orden público y las buenas costumbres, para el intercambio de bienes y servicios o el desarrollo de actividades de cooperación (Corte Constitucional colombiana. Sentencia C-692 de 2007. Magistrado Ponente: Rodrigo Escobar Gil). 


\section{Función disciplinaria del autorregulador del mercado de valores}

La función disciplinaria atribuida al AMV es una manifestación del derecho disciplinario, aplicable a un campo específico del derecho privado, rama que, en sentido amplio, puede entenderse desde distintas perspectivas:

a. Como la disciplina que sujeta a los individuos a una determinada regla de conducta que implica la observancia de leyes y de ordenamientos de una profesión o instituto (Corte Constitucional colombiana. Sentencia C-417 de octubre 4 de 1993. Magistrado Ponente: José Gregorio Hernández Galindo).

b. El derecho disciplinario constituye un derecho-deber que comprende el conjunto de normas, sustanciales y procedimentales, en virtud de las cuales el Estado asegura la obediencia, la disciplina y el comportamiento ético, la moralidad y la eficiencia de los servidores públicos, con miras a asegurar el buen funcionamiento de los diferentes servicios a su cargo. Su finalidad, en consecuencia, es la de salvaguardar la obediencia, la disciplina, la rectitud y la eficiencia de los servidores públicos, y es precisamente allí, en la realización del citado fin, donde se encuentra el fundamento para la responsabilidad disciplinaria, la cual supone la inobservancia de los deberes funcionales de los servidores públicos o de los particulares que ejercen funciones públicas (Corte Constitucional colombiana. Sentencia C-030 de febrero 1 de 2012. Magistrado Ponente: Luis Ernesto Vargas Silva).

En Gómez (2017) el derecho disciplinario está integrado por el derecho disciplinario administrativo, el derecho disciplinario jurisdiccional y el derecho disciplinario delegado. Este último, entendido como aquel que surge de la intervención del Estado en las diferentes profesiones y oficios que pueden ocasionar un riesgo social.

En sentido estricto, la función disciplinaria del AMV es una manifestación del Derecho disciplinario delegado, en tanto impone deberes éticos como mecanismo para regular el comportamiento de quienes ejercen la intermediación en el mercado de valores. En el ejercicio de la función disciplinaria se hace manifiesto el interés del Estado colombiano en el funcionamiento del mercado, no obstante que las transacciones se hacen con capital privado.

Este derecho disciplinario delegado en quien esté autorizado para operar como autorregulador del mercado de valores se caracteriza por:

- Existe un funcionario denominado autorregulador del mercado de valores, a quien se le encarga valorar la inobservancia del ordenamiento jurídico aplicable a intermediarios de valores, así como la omisión o extralimitación en el ejercicio de sus funciones.

- Su finalidad es asegurar el cumplimiento de los deberes de los intermediarios de valores y de sus dependientes.

- Se fundamenta en la diligencia, el cuidado y la corrección en el desempeño de las funciones de intermediarios de valores y del cumplimiento de sus deberes. 
- Las consecuencias jurídicas que se derivan del ejercicio de esta función tienen un carácter sancionatorio, un reproche fundado en la necesidad de castigo a todas aquellas conductas que atenten contra el respeto al orden jurídico que impone deberes a los intermediarios.

- La función disciplinaria forma parte de la esencia de la organización jurídica del mercado de valores y tiene lugar preferente dentro del conjunto de las instituciones jurídicas en la medida en que contribuye a generar confianza en el funcionamiento del mercado.

- Es la manifestación de un poder disciplinario, una facultad atribuida al gremio, al sector de intermediarios del mercado de valores, en virtud del cual un particular llamado autorregulador está legitimado para, con sujeción al marco legal, elaborar un reglamento en el cual se impongan deberes a los intermediarios de valores y a sus dependientes, y para aplicar las sanciones creadas por el Estado colombiano como marco de actuaciones de los miembros de ese sector.

- La función disciplinaria del autorregulador es un mecanismo que concreta los objetivos de intervención estatal en el mercado de valores (artículo 1 Ley 964 de 2005): la protección a los derechos de los inversionistas; promover el desarrollo y la eficiencia del mercado de valores; prevenir y manejar el riesgo sistémico del mercado de valor; y preservar el buen funcionamiento, la equidad, la transparencia, la disciplina y la integridad del mercado de valores y en general la confianza del público en este.

- Al igual que en cualquier proceso disciplinario, se pretende investigar y sancionar las indebidas prácticas en el mercado de valores, es decir, sancionar toda conducta que no esté dentro de los parámetros normativos, con respeto a los lineamientos procesales y principios como debido proceso, proporcionalidad, contradicción, oportunidad, revelación dirigida, economía, celeridad, entre otros.

\section{Proceso disciplinario ante el Autorregulador del Mercado de Valores}

El proceso que adelanta el AMV para imponer sanciones está totalmente reglado, con lo cual se promueve un respeto a las reglas derivadas de la garantía constitucional del debido proceso. El trámite se adelanta de oficio o a petición de parte ante el Tribunal disciplinario del AMV a quien el Estado colombiano le ha dado la potestad disciplinaria sobre los intermediarios de valores, que en lenguaje común son conocidos como comisionistas ${ }^{4}$.

$4 \quad$ El comisionista desarrolla su actividad fundamentado en el contrato comercial de comisión (artículo 1287 Código de Comercio) y para poder participar en el mercado bursátil se le exige estar sometido a la autorregulación definida en la ley, así como a la regulación, supervisión y control estatal en cabeza de la Superintendencia Financiera de Colombia. 
Los comisionistas son intermediarios expertos en transacciones sobre títulos financieros, que ponen a su servicio el conocimiento que tienen de los mercados y el acceso privilegiado de que disfrutan. Bajo esta perspectiva, el comisionista es un asesor experto en evaluar necesidades del inversionista, conoce alternativas existentes en los mercados para responder a ellas de la mejor manera.

El comisionista contribuye a que el emisor de valores satisfaga su interés de obtener financiación, y que el inversionista busque rentabilidad, para ello, se convierte en un facilitador para acceder a un mercado organizado ya bursátil o extrabursátil. En el primer caso, el comisionista de bolsa se define por su pertenencia a una bolsa de valores, en la que actúa a partir de ciertas reglas: i. Hace negocios según la liquidez que ofrecen las bolsas de valores donde participan; ii. Contribuye a la eficiencia de la bolsa y del mercado de valores; iii. Aplica y cumple las reglas y mecanismos de solución de conflictos en transacciones bursátiles; iv. Cumple con los mecanismos de verificación ofrecidos a los clientes sobre las transacciones y, $\mathrm{v}$. Garantiza la seriedad del negocio.

El proceso disciplinario que ejecuta el Tribunal del AMV consta de dos etapas procesales, una de investigación y otra de decisión. Una y otra se explican a continuación:

Figura 1. Etapa de investigación

$\begin{array}{c}\text { Envío de solicitud } \\ \text { formal de } \\ \text { explicaciones }\end{array} \quad$ Explicaciones Pruebas $\left.\quad \ \begin{array}{c}\text { Pliego de cargos } \\ \text { o archivo de la } \\ \text { investigación }\end{array}\right\} \begin{gathered}\text { Traslado de } \\ \text { pliego de } \\ \text { cargos }\end{gathered}$

Fuente: Elaboración Propia.

Con el envio de solicitud formal de explicaciones dirigida al investigado se da inicio al proceso, en ella se le manifiesta de forma clara la conducta por la cual se le investiga, las pruebas que la sustentan y las posibles normas que han sido violadas. Esta solicitud es formulada por el presidente de AMV o el director de Asuntos Legales y Disciplinarios. La normativa que regula este proceso impone que el término para enviar mencionada solicitud al investigado es de tres meses después de la ocurrencia los hechos, en caso de que se trate de una acción realizada por el intermediario; en caso de conductas omisivas, se cuenta a partir del incumplimiento del deber de actuar.

Una vez se hace entrega de la solicitud, el o los investigados deben rendir las explicaciones solicitadas dentro de los diez días hábiles siguientes, término que puede ser ampliado solo por una vez por día, mediante solicitud del investigado en tiempo oportuno ante el presidente de AMV o el director de Asuntos Legales y Disciplinarios. Estas explicaciones puede rendirlas en forma directa o por medio de apoderado en cualquier caso en forma escrita. 
En el escrito de explicaciones se debe manifestar la aceptación o negación de los hechos señalados en la solicitud formal de explicaciones y pronunciarse jurídicamente sobre estos. En caso de que no se emita explicación que niegue los hechos, se tendrá como un indicio grave en contra del investigado.

Vencido el término para rendir explicaciones se inicia la etapa de pruebas. El proceso se caracteriza por conceder libertad probatoria; las pruebas se deben pedir o aportar en el escrito de explicaciones. En el primer caso, el investigado debe especificar qué busca probar con la práctica de esta. En ambos casos, las pruebas se deben decretar y practicar en un término de 20 días hábiles contados a partir del momento en que se decreten.

Practicadas las pruebas el AMV hará una valoración, en consonancia con la solicitud de explicaciones y con las explicaciones dadas por el investigado, lo que le permitirá decidir si formula pliego de cargos o dispone el archivo de la investigación. En caso de que se formule pliego de cargos, quien emitió la solicitud de explicaciones solicita al tribunal disciplinario la imposición de la sanción, y además se corre traslado al investigado, para lo cual se le remite el pliego y se le conceden diez días para que haga los pronunciamientos que considere pertinentes.

Figura 2. Etapa de decisión o juzgamiento

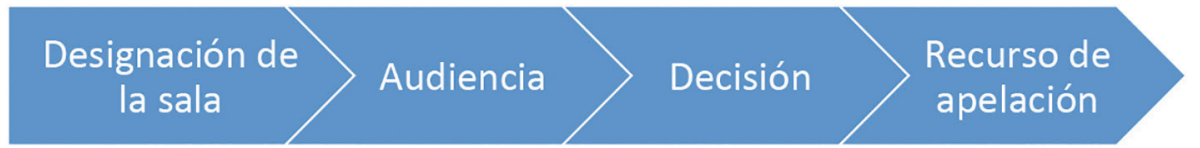

Fuente: Elaboración propia.

La etapa de decisión o de juzgamiento está a cargo del Tribunal Disciplinario, que es un cuerpo colegiado independiente del AMV y está conformado por representantes de la industria de la intermediación en valores y miembros independientes. Una vez el secretario del tribunal recibe el pliego de cargos, se procede a integrar la sala de decisión del tribunal que estará conformado por tres miembros, de los cuales dos son miembros independientes y uno es de la industria ${ }^{5}$, que será la encargada de conocer en primera instancia del proceso.

La sala de decisión tiene la facultad de citar a audiencia al investigado, en la cual se pronunciará mediante la expedición de resoluciones en las que absuelve o sanciona a los investigados, y que quedan notificadas en audiencia. El contenido del pronunciamiento es de carácter público y puede ser consultado en la página web del AMV (https://www.amvcolombia.org.co).

Las sanciones que puede imponer el tribunal al investigado son de diversos tipos: i. Amonestación, entendida como una advertencia severa que hace el Tribunal para que sancionada la conducta no se vuelva a cometer; ii. Multa: La cuantía

5 Incisos 2 y 3 del artículo 42 de los estatutos del AMV. 
máxima aplicable a personas naturales es de 200 salarios mínimos legales vigentes y para personas jurídicas de 1000 salarios de la misma naturaleza; iii. Suspensión: No puede ser inferior a un día hábil ni superior a tres años, y quiere decir que la persona sancionada de esta manera no puede realizar, directa o indirectamente, intermediación en el mercado de valores ni actuar como persona vinculada de un miembro de autorregulado AMV; iv. Expulsión: implica pérdida de la calidad de miembro o asociado autorregulado por el AMV, es decir, se cancela su inscripción como miembro o asociado autorregulado voluntariamente y no podrá realizar actividades de intermediación por 20 años después de que quede en firme la decisión.

Dentro de los ocho días siguientes a la notificación de la decisión se podrá interponer recurso de apelación que será conocido por una sala de revisión de carácter permanente conformada por tres miembros conforme a los estatutos del AMV. Contra la decisión que adopte la sala no se admite recurso.

Finalmente, se destaca que la regulación del proceso contempla una forma de terminación anormal del proceso denominada Acuerdos de Terminación Anticipada - ATA. El investigado y la parte actora, pueden desde la presentación del escrito de explicaciones y hasta antes del fallo, dar por terminado el proceso por medio de un acuerdo en virtud del cual se termine el proceso disciplinario y se acuerde la sanción que se impondrá al investigado. No existe restricción para que este acuerdo se haga en la fase de instrucción o en la fase de juzgamiento, lo que se requiere es que el acuerdo esté firmado por el representante legal del AMV y los investigados.

\section{Función disciplinaria y objetivos de intervención estatal en el mercado de valores}

El marco regulatorio de la función disciplinaria del AMV forma parte del Derecho del Mercado de Valores, disciplina que se ha construido a través de los objetivos de la intervención estatal en el mercado de valores contenidos en la Ley 964 de 2005.

En primer lugar, el Estado colombiano desde la citada ley ha expresado que cualquier disposición legal que se dicte debe estar guiada por la protección de los derechos de los inversionistas. Bajo esta directriz, se impone al gobierno nacional el deber de establecer medidas adecuadas de protección para los inversionistas. En la exposición de motivos de esta ley, se consideró que la principal medida de protección está relacionada con la existencia de mecanismos que propendan por asegurar el flujo de información oportuna, exacta, veraz y suficiente que le permita a los inversionistas conocer los potenciales riesgos y beneficios para tomar decisiones informadas, de manera que se espera que el marco legal deba otorgarles protección contra prácticas engañosas, fraudulentas o de manipulación, así como las relacionadas con el uso y manejo de información privilegiada.

Con la regulación de la función disciplinaria del AMV no se protegen derechos subjetivos del inversionista, los planteamientos formulados muestran que se trata de un proceso en el que se le hace un reproche ético, los resultados del proceso 
disciplinario no tienen como propósito indemnizar perjuicios causados por un comportamiento de los intermediarios. Se trata de un instrumento jurídico a partir del cual el Estado colombiano busca promover o proteger la confianza en el mercado, busca proteger el interés público presente en la actividad bursátil.

En segundo lugar, la ley marco fija como un objetivo de intervención la promoción del desarrollo y la eficiencia del mercado de valores. Con este objetivo se busca que la regulación logre generar el incremento de montos, número de emisiones, número de participantes e impulsar y mejorar el funcionamiento del mercado de valores colombiano. Este objetivo tampoco se cumple con la existencia de una autorregulación regulada, los emisores de valores no encuentran en este marco normativo un incentivo para acudir a los mercados de valores en busca de financiación, en tanto que, si bien necesitan de un intermediario para ofrecer sus valores en el mercado, la relación contractual que surge entre ellos y el intermediario está fuera del alcance de las conductas que el marco normativo de la autorregulación reprocha desde el punto de vista ético.

La función disciplinaria del AMV, en el plano teórico, guarda directa relación con el objetivo de intervención que la ley colombiana enuncia como la preservación del buen funcionamiento, la equidad, la transparencia, la disciplina y la integridad del mercado de valores y en general la confianza del público en este. Bajo esta directriz, la regulación y supervisión debe concentrarse en la definición, detección y sanción de prácticas desleales de negociación y las conductas contrarias al mercado, bajo el entendido que los mercados de capitales se basan en la confianza del público y la disciplina del mercado, lo que los hace vulnerables a comportamientos irracionales y pánico en los inversionistas.

Castaño (2011) reconoce que existe dificultad en establecer criterios objetivos que posibiliten medir la eficiencia en el funcionamiento de un esquema de regulación, acepta que tanto el modelo de autorregulación como la forma en que el AMV desarrolla sus funciones ha permitido avanzar de manera eficiente en la consolidación y fortalecimiento de la transparencia, integridad y profesionalismo en el mercado de valores colombiano.

Si bien, la función disciplinaria del AMV no tiene un carácter indemnizatorio, sí produce como efecto un castigo reputacional en los intermediarios de bolsa, un castigo reputacional que no protege al inversionista, que solo le sirve para saber si negocia con operadores autorizados. Se requiere hacer un ajuste al marco normativo del AMV, lo deseable es que se centre atención en el gobierno corporativo, de manera que lo integren personas conocedoras del mercado, pero no vinculadas con los mismos investigados; de igual manera, debe revisarse su obligatoriedad, sería prudente pensar que si el intermediario acepta de manera voluntaria someterse a él es porque tiene claro que es un sello de calidad.

En realidad, la creación del AMV con diversas funciones más que a objetivos de intervención estatal guiados por la protección a derechos de inversionistas, solo pone en evidencia unos límites a las posibilidades de actuación y delimita 
las posibilidades de actuación de quienes conocen el mercado, su funcionamiento, los riesgos en espacios organizados de negociación de valores, que se enmarcan en lo que Ibáñez (2002) denomina eticidad y parámetros éticos, integrados por estándares que forman parte de una ética profesional en el mercado, orientados a fomentar la actividad transparente de todos los operadores de mercado.

Una protección institucional adecuada para los inversionistas, sujetos sin los cuales no funcionaría el mercado de valores, sería el diseñado por North (2001), aquel que disminuye o elimina los costos de transacción, asegura el cumplimiento de los contratos y soluciona las asimetrías de información. Bajo esta directriz, la autorregulación del mercado de valores no puede ser el instrumento adecuado para garantizar derechos a inversionistas.

\section{CONCLUSIONES}

Con la autorregulación en el mercado de valores el Estado colombiano no renuncia ni delega la función legislativa, las normas provenientes de autorregulación cualquiera que sea su manifestación son un complemento de la normativa estatal, y constituyen un límite para la actuación de los intermediarios. Bajo esta perspectiva, se reconoce que tanto la normativa estatal como la proveniente de los particulares, de manera aislada, son insuficientes para cumplir los propósitos de la intervención estatal en los mercados, así las cosas, no compartimos que en países como Colombia la adopción de esquemas de autorregulación ha estado acompañada del debilitamiento o abandono del papel regulador del Estado (Rodríguez, 2014), el Estado no se debilita, es un Estado que promueve una autorregulación regulada, tampoco entrega el control a los particulares para ejercer una función inherente a la del papel del Estado que es la función legislativa.

Los problemas que pueden surgir de este modelo de autorregulación se concretan en la existencia de múltiples regulaciones que podrían traer conflictos de contenido normativo, conflictos de competencias, que bajo la justificación de ser un instrumento que esté guiado por los propósitos de la intervención estatal en el mercado muestre a los intermediarios campos no regulados donde podrían desarrollar sus actividades sin límite, donde su creatividad para buscar la satisfacción de su interés individual opere sin restricciones legales.

Consideramos que para que la autorregulación produzca los efectos que una perspectiva teórica o práctica pueda identificar, es necesario centrar atención en los siguientes aspectos: i. ¿En qué asuntos es pertinente abrir espacio para la autorregulación? ii. ¿Quién o quiénes son los encargados de autorregular? iii. Si autorregula el mismo gremio ¿cómo garantizar la imparcialidad a la hora de ejercer su función disciplinaria?; iv. Al no pertenecer a la organización estatal ¿Qué incentivos o motivaciones tiene el autorregulador del mercado para ejercer sus funciones, en especial, la disciplinaria? y v. ¿Existe mecanismos para proteger al mercado, a los inversionistas y a intermediarios de errores y omisiones del autorregulador? 
Aun cuando la autorregulación en los mercados de valores sea una exigencia legal, ella no es un instrumento para la protección de derechos patrimoniales, su existencia se justifica solo como un medio de protección de la reputación en la oferta de bienes y servicios, reputación que interesa al autorregulador como a los agentes regulados que no siempre va a coincidir con la intención estatal, dado que el interés del agente regulado es un interés individual, y el interés del Estado se enfoca más a la protección del interés general y del mercado.

Otro tema que es objeto de análisis es el relacionado con las sanciones que puede imponer el autorregulador, reconocemos que es posible que la sanción que se imponga no es lo suficientemente fuerte o que tenga la potencialidad de generar un impacto real, tanto en el intermediario, objeto de la sanción disciplinaria, como en el mercado. El modelo de regulación y la forma de funcionamiento del mercado no están diseñados para generar un modelo de promover la realización de determinada conducta a partir del rigor de una sanción, ellos están diseñados para recordar a los intermediarios sus deberes legales y éticos que puedan promover confianza en el mercado, que tengan la posibilidad de identificar y administrar riesgos actuales y potenciales del mercado derivados de la conducta de sus agentes y de los esquemas de negocios.

Cualquier regulación, la estatal o la particular, o ambas no tendrán la potencialidad de extinguir los riesgos del mercado de valores que cada día son mayores, máxime si a ello sumamos el entorno digital en que tiene lugar la negociación de valores, necesario es aceptar que el mercado de valores tiene una alta capacidad para generar riesgo sistémico, esto trae consigo el reconocer la insuficiencia de la regulación pública y privada para contrarrestar riesgos, y el aceptar que no basta con la existencia de mecanismos que permitan identificar acciones, procedimientos y sanciones, se requiere que el intermediario valore la confianza que en él deposita el inversionista y que el este conozca los riesgos que asume en cualquier negociación.

\section{REFERENCIAS}

Ariza, E. (2012). Autorregulación y debido proceso. Bogotá, Colombia: Ibáñez.

Black, J.(1996, enero). Constitutionalising Self-regulation. Recuperadoel 2 de septiembre de 2019, de https://onlinelibrary.wiley.com/doi/abs/10.1111/j.1468-2230.1996. tb02064.X

Castaño, J. (2011). Autorregulación y autorregulador: cómo el autorregulador contribuye a la construcción de un marco institucional eficiente en el mercado de valores colombiano. En C. Blanco (Ed.). Cinco años de la Ley 964 de 2005 (pp. 129-156). Bogotá, Colombia: Universidad Externado de Colombia.

Gómez, C. (2017). Dogmática del derecho disciplinario. Bogotá, Colombia: Universidad Externado de Colombia. 
Ibañez, J. (2002). Ética y normas de conducta profesional en el mercado de valores. En J. Wenceslao (Ed.). Ética y mercado de valores (pp. 25-62). Madrid, España: Marcial Pons.

Larrañaga, P. (2009). Regulación técnica jurídica y razonamiento económico. México: Porrúa.

North, D. (2001). Instituciones, cambio institucional y desempeño económico. México: Fondo de Cultura Económica.

Rodríguez, M. (2014). Concepto, alcance y estructura de la autorregulación en el mercado de valores colombiano. Revista E-Mercatoria, 13(2), 29-96. Recuperado de https://revistas.uexternado.edu.co/index.php/emerca/article/view/4047

Zimmerman, B.J. (2000). Attaining self-regulation: A social cognitive perspective. En M. Boekaerts, P.R. Pintrich y M. Zeidner (Eds.). Handbook of selfregulation (pp. 13-40).

\section{Jurisprudencia citada}

Corte Constitucional colombiana. Sentencia C-417 de octubre 4 de 1993. Magistrado Ponente: José Gregorio Hernández Galindo.

Corte Constitucional colombiana. Sentencia C-692 de 2007. Magistrado Ponente: Rodrigo Escobar Gil.

Corte Constitucional colombiana. Sentencia C-030 de febrero 1 de 2012. Magistrado Ponente: Luis Ernesto Vargas Silva.

\section{Legislación colombiana}

Constitución Política de Colombia 1991

Ley 964 de 2005

Decreto 2555 de 2010

Reglamento del Autorregulador del Mercado de Valores Colombiano 